\title{
Repetibilidade da produção de sementes em clones de guaraná
}

\author{
Firmino José do Nascimento Filho(1), André Luiz Atroch ${ }^{(1)}$, Cosme Damião Cruz(2) \\ e Pedro Crescêncio Souza Carneiro(2)
}

\begin{abstract}
(1)Embrapa Amazônia Ocidental, Caixa Postal 319, CEP 69010-970 Manaus, AM. E-mail: firmino.filho@cpaa.embrapa.br, andre.atroch@cpaa.embrapa.br (2)Universidade Federal de Viçosa, Departamento de Biologia Geral, Bioagro, Avenida P.H. Rolfs, s/no, CEP 36571-000 Viçosa, MG. E-mail: cdcruz@ufv.br, carneiro@ufv.br
\end{abstract}

Resumo - O objetivo deste trabalho foi avaliar a repetibilidade da produção de sementes de 27 clones de guaraná (Paullinia cupana). Os clones foram avaliados em dez ensaios instalados em blocos ao acaso com duas repetições e três plantas por parcela, com espaçamento de $5 \times 5 \mathrm{~m}$. A produção de sementes foi avaliada por quatro anos $(1998,1999,2000$ e 2001) a partir do início da fase produtiva, após dois anos do plantio. O coeficiente de repetibilidade foi estimado pelos métodos: análise de variância (ANOVA), componentes principais com base em matriz de correlações (CPCOR), componentes principais com base em matriz de variâncias e covariâncias fenotípicas (CPCOV) e análise estrutural com base na matriz de correlações (AECOR). Foram estimados também o número mínimo de observações e o coeficiente de determinação. As estimativas dos coeficientes de repetibilidade com base nos quatro anos, nos dez ambientes, foram relativamente baixas - 0,2618 (AECOR), 0,3064 (CPCOR), 0,3183 (ANOVA) e 0,5308 (CPCOV) - e variaram de acordo com o método e o ambiente. São necessários, no mínimo, quatro anos de avaliação para a obtenção de valores genotípicos dos clones com coeficiente de determinação de $80 \%$.

Termos para indexação: Paullinia cupana, análise de variância, análise estrutural, componente principal, seleção clonal.

\section{Seed yield repeatability of guarana clones}

\begin{abstract}
The aim of this work was to evaluate the seed yield repeatability of 27 guarana clones. Ten assays were carried out for clone evaluation using a completely randomized block design, with two replicates and three plants per plot with a $5 \times 5 \mathrm{~m}$ spacing. The seed yield was measured during four years $(1998,1999,2000$ and 2001) after the beginning of the production phase, two years after planting. Four methods were used for repeatability coefficient estimation: analysis of variance (ANOVA), principal components analysis based on correlation matrix (CPCOR), principal components analysis based on phenotypical variance and covariance matrix (CPCOV), and structural analysis based on correlation matrix (AECOR). Minimum observations number and determination coefficient were also evaluated. The repeatability coefficient estimates based on these four years, in all ten environments, were relatively low -0.2618 (AECOR), 0.3064 (CPCOR), 0.3183 (ANOVA) and $0.5308(\mathrm{CPCOV})$ - and varied according to method and environment. At least four years of evaluation are necessary for obtaining the real genotypical value of the clones for an $80 \%$ determination coefficient.
\end{abstract}

Index terms: Paullinia cupana, analysis of variance, structural analysis, principal component, clone selection.

\section{Introdução}

O coeficiente de repetibilidade é uma característica que, segundo Lush (1964), Abeywardena (1972), Kempthorne(1973) eCruzetal. (2004), pode ser definida em termos estatísticos como a correlação entre as medidas feitas em um mesmo indivíduo sob variações no tempo ou no espaço. Para Chapman (1985), esse coeficiente representa a proporção da variância fenotípica total de um caráter que é explicada por diferenças permanentes entre indivíduos. Essas diferenças são ocasionadas por variações nos genótipos e pelas alterações permanentes atribuídas ao ambiente comum. De acordo com Falconer (1989), não apenas as diferenças permanentes entre indivíduos, mas também as diferenças causadas pelo ambiente temporário contribuem para a variância fenotípica total. Essa variância só poderá ser analisada em termos de variância dentro de indivíduos e de variância entre indivíduos quando forem efetuadas várias medidas de um mesmo caráter em cada indivíduo. 
O coeficiente de repetibilidade representa o valor máximo que a herdabilidade em sentido amplo pode atingir. Quando a variância proporcionada pelos efeitos permanentes do ambiente é minimizada, a repetibilidade aproxima-se da estimativa da herdabilidade (Cruz et al., 2004). Se o valor da estimativa de repetibilidade for alto, a seleção com base em apenas uma ou em poucas observações será eficiente e, se o valor da estimativa for baixo, será necessário calcular a média de várias observações para alcançar a mesma eficiência de seleção (Lush, 1964; Turner \& Young, 1969). O coeficiente de repetibilidade permite determinar o número de medições necessárias que devem ser feitas em cada indivíduo ao longo do tempo, para que haja eficiência na seleção fenotípica realizada entre os genótipos, o que pode resultar na redução de custos e de trabalho na experimentação (Cornacchia et al., 1995; Pereira et al., 1998; Ferreira et al., 1999; Cruz et al., 2004). Para Falconer (1981) e Cruz et al. (2004), também é necessário levar em consideração que as estimativas de repetibilidade variam de acordo com a natureza da característica avaliada, com as propriedades genéticas da população e com as condições em que os indivíduos se desenvolvem.

Caso o genótipo do indivíduo em que se realizam as medidas repetidas não se encontre estabilizado, Cruz et al. (2004) chamam a atenção para três aspectos que devem ser considerados: a variação dentro de indivíduos inclui uma porção considerável da variância da interação do genótipo com os efeitos temporários do ambiente; o aumento do número de repetição de medidas com a finalidade de reduzir essa variação pode não ser vantajoso, pois a variância adicional proporcionada pela interação entre genótipos e o ambiente temporário pode ser suficiente para neutralizar aquela redução; as expressões descritas na literatura para o cálculo da repetibilidade podem não ter validade.

Estudos de repetibilidade já foram aplicados ao melhoramento de diversas culturas perenes, como cajazeira(Soares etal., 2008), laranjeira-doce(Negreiros et al., 2008), dendê (Cedillo, 2003), café (Bonomo, 2002), aceroleira (Lopes et al., 2001), cajueiro-anão (Cavalcanti et al., 2000), cacaueiro (Dias \& Sousa, 1993; Carvalho, 1999), cupuaçuzeiro (Costa et al., 1997), erva-mate (Resende et al., 1995), seringueira (Gonçalves et al., 1990) e coqueiro (Siqueira, 1982). Em guaranazeiro, Vallois et al. (1979) avaliaram as seguintes características em estudo de repetibilidade: tamanho da inflorescência, número de botões florais e número de frutos por inflorescência e de sementes por frutos.

O objetivo deste trabalho foi avaliar a repetibilidade da produção de sementes de 27 clones de guaraná.

\section{Material e Métodos}

Em 1996, foi implantada em campos experimentais da Embrapa Amazônia Ocidental uma rede de ensaios para a avaliação de 27 clones de guaraná, constituída por dez experimentos instalados em três municípios do Estado do Amazonas (Maués, Manaus e Iranduba), em três tipos de ecossistema (mata primária, mata secundária e capoeira) e com duas condições de cultivo (com e sem adubação), formando os seguintes ambientes: 1, Iranduba, mata secundária, com adubação; 2, Iranduba, mata secundária, sem adubação; 3, Manaus, mata secundária, com adubação; 4, Manaus, mata secundária, sem adubação; 5, Manaus, capoeira, com adubação; 6, Manaus, capoeira, sem adubação; 7, Maués, mata primária, com adubação; 8 , Maués, mata primária, sem adubação; 9, Maués, capoeira, com adubação; 10, Maués, capoeira, sem adubação. O delineamento utilizado em todos os ensaios foi o de blocos ao acaso, com duas repetições e três plantas por parcela, com espaçamento de $5 \times 5 \mathrm{~m}$.

A partir do segundo ano após o plantio, na fase produtiva, foi avaliada a produção de sementes secas por planta. Essa avaliação foi feita com base na massa da biomassa fresca dos frutos maduros. Essa massa incluiu a ráquis (parte central do cacho) e as sementes com arilo. Para a obtenção apenas da massa das sementes secas, foi feita a conversão da massa de toda aquela biomassa por meio da relação 6:1 (Smyth \& Cravo, 1989). A variável produção, correspondente à massa de sementes secas por planta, em gramas, foi avaliada durante quatro anos consecutivos: 1998-2001.

Para estimar com maior consistência o coeficiente de repetibilidade (r), foram utilizados quatro procedimentos: análise de variância (ANOVA), análise dos componentes principais com base na matriz de correlações (CPCOR), análise dos componentes principais com base na matriz de variâncias e covariâncias fenotípicas (CPCOV) e análise estrutural com base na matriz de correlações ( $r$ médio - AECOR).

$\mathrm{Na}$ ANOVA, o modelo estatístico adotado, que considera dois fatores de variação, 
foi $Y_{i j}=\mu+g_{i}+a_{j}+\varepsilon_{i j}$, em que $Y_{i j}$ é a observação referente ao i-ésimo clone de guaraná, no j-ésimo ano; $\mu$ é a média geral; $g_{i}$ é o efeito aleatório do i-ésimo clone, sob a influência do ambiente permanente $g_{i} \sim$ NID $\left(0, \sigma_{\mathrm{p}}^{2}\right) ; \mathrm{a}_{\mathrm{j}}$ é o efeito fixo do ambiente temporário na j-ésima medição $\sum_{\mathrm{j}}^{\mathrm{n}} \mathrm{a}_{\mathrm{j}}=0$; e $\varepsilon_{\mathrm{ij}}$ é o erro experimental estabelecido pelos efeitos temporários do ambiente na j-ésima medição do i-ésimo genótipo $\left[\varepsilon_{\mathrm{ij}} \sim \mathrm{NID}\left(0, \sigma^{2}\right)\right]$. Com base nesse modelo, tem-se o esquema de análise de variância apresentado na Tabela 1 .

Com base no modelo reduzido, e considerando $\mathrm{Y}_{\mathrm{ij}}$ e $\mathrm{Y}_{\mathrm{ij}}$ como as diferentes medidas realizadas num mesmo indivíduo em diferentes anos, o coeficiente de repetibilidade é dado por:

$\mathrm{r}=\frac{\operatorname{Côv}\left(\mathrm{Y}_{\mathrm{ij}}, \mathrm{Y}_{\mathrm{ij}}\right)}{\sqrt{\hat{\mathrm{V}}\left(\mathrm{Y}_{\mathrm{ij}}\right) \cdot \hat{\mathrm{V}}\left(\mathrm{Y}_{\mathrm{ij}}\right)}}=\frac{\hat{\sigma}_{\mathrm{p}}^{2}}{\hat{\sigma}^{2}+\hat{\sigma}_{\mathrm{p}}^{2}}=\frac{\mathrm{QMG}-\mathrm{QMR}}{\mathrm{QMG}+(\mathrm{n}-1) \mathrm{QMR}}$, em que $\hat{\sigma}_{\mathrm{p}}^{2}$ é a variabilidade genética acrescida da variação proporcionada pelos efeitos permanentes do ambiente.

O método dos componentes principais com base na matriz de correlações (CPCOR) consiste na obtenção de uma matriz de correlação entre os clones, em cada par de medições. São determinados, na matriz, os autovalores e os respectivos autovetores normalizados associados. $\mathrm{O}$ autovetor, cujos elementos apresentam mesmo sinal e magnitudes próximas, é aquele que expressa a tendência dos clones em manter, ao longo dos anos, suas posições relativas em relação aos demais (Abeywardena, 1972; Cruz et al., 2004). A proporção do autovalor associado a esse autovetor é o estimador do coeficiente de repetibilidade, ou seja: $r=\frac{\hat{\lambda}_{k}}{\sum_{j=k}^{n} \hat{\lambda}_{j}}$, $(\mathrm{j}=1,2, \ldots, \mathrm{n})$, em que $\mathrm{n}$ é o número de medições realizadas; $\hat{\lambda}_{\mathrm{k}}$ é o autovalor associado ao autovetor, cujos elementos têm o mesmo sinal e magnitudes semelhantes.

Segundo Rutledge (1974), se o coeficiente de repetibilidade for estimado da forma descrita anteriormente, ele será influenciado, indevidamente pelo número de medições realizadas. Esse autor considera

Tabela 1. Esquema da análise de variância com dois fatores de variação para o estudo de repetibilidade.

\begin{tabular}{lllc}
\hline Fonte de avaliação & \multicolumn{1}{c}{ GL } & QM & E $(\mathrm{QM})^{(1)}$ \\
\hline Anos & $\mathrm{n}-1=3$ & QMA & - \\
Genótipos & $\mathrm{g}-1=26$ & QMG & $\sigma^{2}+\mathrm{n}_{\mathrm{p}}^{2}$ \\
Resíduo & $(\mathrm{g}-1)(\mathrm{n}-1)=78$ & QMR & $\sigma^{2}$ \\
\multicolumn{2}{l}{${ }^{(1)} \sigma^{2}$, componente de variância devido ao erro; $\sigma_{\mathrm{p}}^{2}$, componente de variância } \\
\multicolumn{4}{l}{ devido aos genótipos. }
\end{tabular}
devido aos genótipos. que o estimador de $\mathrm{r}$ apresentado a seguir é mais adequado:

$\mathrm{r}=\frac{\hat{\lambda}_{1}-1}{\mathrm{n}-1}$.

Para o método dos componentes principais CPCOV, o estimador da repetibilidade é dado por: $\mathrm{r}=\frac{\hat{\lambda}_{1}-\hat{\sigma}_{\mathrm{Y}}^{2}}{\hat{\sigma}_{\mathrm{Y}}^{2}(\mathrm{n}-1)}$, em que: $\hat{\sigma}_{\mathrm{Y}}^{2}=\frac{1}{\mathrm{n}} \sum_{\mathrm{j}=1}^{\mathrm{n}} \hat{\sigma}_{\mathrm{j}}^{2}=\hat{\sigma}^{2}+\hat{\sigma}_{\mathrm{p}}^{2}$.

A obtenção do coeficiente de repetibilidade mediante o método da análise estrutural ( $\mathrm{r}$ médio, AECOR) apresenta apenas diferenças conceituais em relação aos métodos baseados nos componentes principais. Mansour et al. (1981), autores deste método, consideram $\mathrm{R}$ a matriz paramétrica de correlações entre tratamentos em cada par de avaliações e $\hat{R}$, o seu estimador. Um estimador do coeficiente de repetibilidade baseado nos componentes principais é: $\mathrm{r}=\frac{\hat{\lambda}_{1}-1}{\mathrm{n}-1}=\frac{\alpha^{\prime} \hat{\mathrm{R}} \alpha-1}{\mathrm{n}-1}$, em que $\hat{\lambda}_{1}=\hat{\alpha}^{\prime} \hat{\mathrm{R}} \alpha$ é o maior autovalor de $\hat{\mathrm{R}}$ e $\hat{\alpha}^{\prime}=\left\{\mathrm{a}_{1}, \mathrm{a}_{2}, \ldots . \mathrm{a}_{\mathrm{n}}\right\}$ é o autovetor associado a $\hat{\lambda}_{1}$, tal que $\hat{\alpha}^{\prime} \alpha=1$, ou seja, o autovetor está normalizado.

Segundo Morrison (1967), o autovetor cujos elementos têm mesmo sinal e magnitudes semelhantes e que está associado ao maior autovalor $\left(\hat{\lambda}_{1}\right)$ de $\mathrm{R}$ é

expresso por: $\alpha^{\prime}\left\{\frac{1}{\sqrt{\mathrm{n}}}, \cdots, \frac{1}{\sqrt{\mathrm{n}}}\right\}$.

Utilizando-se o autovetor $\alpha$, que segundo Mansour et al. (1981) corresponde ao maior autovalor de $\mathrm{R}$, o estimador do coeficiente de repetibilidade com base na análise estrutural é dado por: $r=\frac{\alpha^{\prime} \hat{R} \alpha-1}{n-1}=\frac{2}{n(n-1)} \sum_{j=1}^{n} \sum_{j^{\prime}=2}^{n} \hat{\rho}_{j j^{\prime}}$, com $j<j$, em que $\hat{\rho}_{\mathrm{ji}}$ é o elemento da j-ésima linha e j'-ésima coluna da matriz $\hat{\mathrm{R}}$.

Assim, esse estimador do coeficiente de repetibilidade é a média aritmética das correlações fenotípicas entre genótipos, considerando cada par de anos.

Uma vez obtido o coeficiente de repetibilidade, o número mínimo de medições que devem ser realizadas para se predizer o valor real dos indivíduos com base em um coeficiente de determinação $\left(\mathrm{R}^{2}\right)$ pre-estabelecido foi determinado. 
A predição desse valor foi realizada com base na expressão (Lush, 1964): $\mathrm{n}_{\mathrm{m}}=\frac{\mathrm{R}^{2}(1-\mathrm{r})}{\left(1-\mathrm{R}^{2}\right) \mathrm{r}}$, em que: $\mathrm{n}_{\mathrm{m}}$ é o número de medições necessárias para predição do valor real; $\mathrm{R}^{2}$ é o coeficiente de determinação pre-estabelecido; e $\mathrm{r}$, o coeficiente de repetibilidade obtido por um dos métodos utilizados.

$\mathrm{O}$ coeficiente de determinação genotípico $\left(\mathrm{R}^{2}\right)$, que representa a certeza da predição do valor real dos clones selecionados com base em $\mathrm{n}$ medições realizadas, foi obtido pela expressão: $\mathrm{R}^{2}=\frac{\mathrm{nr}}{1+\mathrm{r}(\mathrm{n}-1)}$, em que: $\mathrm{R}^{2}$ é o coeficiente de determinação genotípico; $\mathrm{n}$ é o número de medições realizadas ( $\mathrm{n}=4$ neste estudo); e r, o coeficiente de repetibilidade.

Todas as análises estatísticas foram feitas com o programa computacional GENES (Cruz, 2001).

\section{Resultados e Discussão}

Foram detectadas diferenças significativas entre os clones para a característica produção de sementes secas por planta, exceto no ambiente 6 , o que é indicativo da existência de heterogeneidade no material genético e de possibilidade de êxito com a seleção dos genótipos mais promissores (Tabela 2).

A produção média de sementes secas variou de 260 a 765 g por planta (Tabela 2), com os coeficientes de variação entre 53 e $73 \%$ que, segundo Atroch \& Nascimento Filho (2005), são considerados de média precisão. Nascimento Filho et al. (2000a), ao analisar dados de 13 experimentos de competição de clones de guaranazeiro avaliados num período que variou de quatro a nove anos consecutivos, obtiveram coeficiente de variação médio de $74 \%$ para as análises individuais e de $92 \%$ para a análise conjunta.

Bonomo (2002), em estudos de progênies de cafeeiros, relatou que, além das diferenças nas condições das mudas e na necessidade de replantios, as altas magnitudes dos coeficientes de variação experimental também podem estar associadas a pequenas diferenças nos tratos culturais aplicados à cultura.

As correlações entre as produções anuais de sementes secas, em gramas por planta, nos dez ambientes estão apresentadas na Tabela 3. As correlações foram de baixa magnitude ou não significativas. A correlação entre uma mesma característica avaliada em dois períodos, além de refletir associação de natureza genética e ambiental, mede indiretamente a interação genótipos x ambientes (Ferreira et al., 1999). No presente trabalho, a falta de correlação indica, indiretamente, a existência da interação genótipos x ambientes, ou seja, o comportamento diferenciado dos clones de um ano para o outro. Essa observação confirma os resultados encontrados por Nascimento Filho et al. (2000a) em experimentos de avaliação de clones quanto à produção, nos quais foram detectados efeitos significativos da interação clones $\mathrm{x}$ anos. Em avaliação de cultivares de alfafa com base em caracteres forrageiros, Ferreira et al. (1999) encontraram resultados semelhantes.

Apesar da baixa correlação manifestada na maioria dos pares de anos considerados, foi possível constatar que houve maior consistência nas medições referentes às duas últimas produções, representadas pelos anos 2000 e 2001, conforme verificado nos ambientes 4, 7, 8,9 e 10. No ambiente 8, por exemplo, essa correlação foi de alta magnitude (Tabela 3 ).

Escobar et al. (1984), ao avaliar a produção de sementes secas de plantas de guaranazeiro de pé-franco

Tabela 2. Quadrado médio das análises de variância e média da produção de sementes secas por planta, de clones de guaraná testados em solos caracterizados pelo tipo de vegetação capoeira, mata primária e mata secundária, em dois sistemas de cultivo - com e sem o uso de adubação - avaliados em diferentes ambientes durante quatro anos consecutivos (1998, 1999, 2000 e 2001) nos municípios de Manaus, Maués e Iranduba, no Estado do Amazonas.

\begin{tabular}{|c|c|c|c|c|c|c|c|c|c|c|c|}
\hline \multirow[t]{2}{*}{ Fonte de variação } & \multirow[t]{2}{*}{ GL } & \multicolumn{10}{|c|}{ Ambientes $^{(1)}$} \\
\hline & & 1 & 2 & 3 & 4 & 5 & 6 & 7 & 8 & 9 & 10 \\
\hline Ano & 3 & 3.326 .954 & 729.842 & 1.075 .788 & 3.343 .406 & 223.471 & 625.714 & 647.317 & 1.829 .920 & 1.488 .469 & 810.298 \\
\hline Clone & 26 & $306.775^{*}$ & $190.043 * *$ & * $139.021 * *$ & $304.145^{*}$ & $377.893 * *$ & $124.510^{\mathrm{ns}}$ & $520.538 * *$ & $386.227 * *$ & $423.183^{* *}$ & $69.733^{* *}$ \\
\hline Resíduo & 78 & 167.159 & 81.129 & 58.129 & 158.430 & 122.621 & 96.099 & 169.283 & 130.023 & 111.847 & 29.707 \\
\hline Média (g por planta & & 557 & 475 & 435 & 594 & 539 & 426 & 765 & 550 & 576 & 260 \\
\hline $\mathrm{CV}(\%)$ & & 73 & 59 & 55 & 66 & 64 & 72 & 53 & 65 & 58 & 66 \\
\hline
\end{tabular}


por seis anos, verificaram que as correlações com a média geral entre médias acumuladas de ano em ano ou de médias bianuais dos seis anos aumentaram com a idade das plantas e concluíram que a avaliação com base nos três primeiros anos é suficiente para estimar, com boa precisão, a produção média dos seis anos. Nesse mesmo estudo, os autores verificaram que as correlações relativas às associações da produção do primeiro ano com o segundo, do segundo com o terceiro, e assim sucessivamente até o quinto ano, aumentaram. Esse aumento na magnitude das correlações se dá em virtude do aumento do número de plantas que vão se tornando produtivas ao longo dos anos, uma vez que, em mudas oriundas de sementes, a estabilidade produtiva ocorre em torno do quinto ano de produção.

O início da estabilização da produção foi evidenciado nos ambientes 7,8 e 9 , relativos a Maués, onde os clones expressaram seu maior potencial produtivo (Tabela 2). A análise da Tabela 3 demonstra que os valores das correlações entre os anos de 2000 e 2001 foram de alta magnitude e significativos. Esse resultado corrobora a estabilização da produção observada em 1999, quando as correlações entre 1999 e 2000 também foram significativas, embora de baixa magnitude. Nascimento Filho et al. (2000b) observaram que o processo de estabilização da produção de clones de guaranazeiro inicia-se após o terceiro ano do plantio, caso não haja nenhum efeito climático que interfira negativamente no desenvolvimento geral das plantas.

Emboranão existam, na literatura, estudos específicos de repetibilidade que envolvam a característica de produção de sementes secas por planta de clones de guaraná, Escobar (1986), ao considerar o fato de que o guaranazeiro é, por natureza, uma espécie altamente heterogênea, prevê que as variações entre plantas, clones e anos serão sempre maiores nos primeiros anos de colheitas e conclui que provavelmente sejam necessários vários anos de acompanhamento da produção para quantificar, com precisão, o potencial produtivo de clones de guaraná. Esse fato é confirmado pela correlação intraclasse da combinação de anos para a variável produção (Tabela 3), na qual se verifica que as magnitudes das correlações no primeiro ano de produção, 1998, foram muito baixas.

As estimativas de repetibilidade obtidas, de modo geral, foram relativamente baixas e variaram de acordo com as metodologias utilizadas e com os ambientes onde os clones foram testados (Tabela 4).

A média encontrada para as estimativas dos coeficientes de repetibilidade e de determinação com base nos quatro anos de avaliação foi de $0,3543 \mathrm{e}$ $63,14 \%$, respectivamente, o que indica serem necessárias várias medições para se acessar o valor genotípico dos clones de guaraná (Tabela 4). As baixas estimativas do coeficiente de repetibilidade implicam dificuldades para o melhorista em identificar os melhores materiais genéticos a partir de poucas medições (Ferreira et al., 1999).

Entre os métodos utilizados, o CPCOV foi o que apresentou os maiores valores de coeficiente de repetibilidade $(0,5308$, Tabela 4$)$. Os baixos valores do coeficiente de repetibilidade obtidos com a ANOVA podem ser explicados pelos altos valores do quadrado médio do resíduo, evidenciados pelos elevados coeficientes de variação experimental (Tabela 2). Para os métodos CPCOR e AECOR, os baixos valores dos coeficientes de repetibilidade (Tabela 4) podem estar relacionados, em parte, às baixas correlações

Tabela 3. Coeficientes de correlação intraclasse da combinação de anos da variável produção de sementes secas por planta, de clones de guaraná testados em solos caracterizados pelo tipo de vegetação capoeira, mata primária e mata secundária, em dois sistemas de cultivo - com e sem o uso de adubação - avaliados em diferentes ambientes durante quatro anos consecutivos (1998, 1999, 2000 e 2001) nos municípios de Manaus, Maués e Iranduba, no Estado do Amazonas.

\begin{tabular}{|c|c|c|c|c|c|c|c|c|c|c|}
\hline \multirow[t]{2}{*}{ Pares de anos } & \multicolumn{10}{|c|}{ Ambientes $^{(1)}$} \\
\hline & 1 & 2 & 3 & 4 & 5 & 6 & 7 & 8 & 9 & 10 \\
\hline 1998-1999 & $0,5854^{* *}$ & $0,5917^{* *}$ & $0,3416^{\mathrm{ns}}$ & $0,0900^{\text {ns }}$ & $0,3156^{\mathrm{ns}}$ & $-0,0396^{\mathrm{ns}}$ & $0,5648^{* *}$ & $0,2892^{\mathrm{ns}}$ & $0,1316^{\mathrm{ns}}$ & $0,3508^{\mathrm{ns}}$ \\
\hline $1998-2000$ & $0,0343^{\text {ns }}$ & $0,3670^{\mathrm{ns}}$ & $0,3905^{*}$ & $0,2055^{\mathrm{ns}}$ & $0,1888^{\text {ns }}$ & $-0,1057^{\mathrm{ns}}$ & $0,2388^{\mathrm{ns}}$ & $-0,0044^{\mathrm{ns}}$ & $-0,0016^{\mathrm{ns}}$ & $0,3575^{\mathrm{ns}}$ \\
\hline $1999-2000$ & $0,0645^{\mathrm{ns}}$ & $0,5517^{* *}$ & $0,4455^{*}$ & $-0,1829^{\mathrm{ns}}$ & $0,5283^{* *}$ & $0,2792^{\mathrm{ns}}$ & $0,3763^{\mathrm{ns}}$ & $0,3358^{\mathrm{ns}}$ & $0,6486^{* *}$ & $0,4349^{*}$ \\
\hline $1999-2001$ & $0,4095^{*}$ & $-0,0223^{\mathrm{ns}}$ & $0,1961^{\mathrm{ns}}$ & $-0,1073^{\mathrm{ns}}$ & $0,4630^{*}$ & $0,1954^{\mathrm{ns}}$ & $0,1821^{\mathrm{ns}}$ & $0,1751^{\mathrm{ns}}$ & $0,6195^{* *}$ & $0,1116^{\mathrm{ns}}$ \\
\hline 2000-2001 & $0,2386^{\mathrm{ns}}$ & $0,2765^{\mathrm{ns}}$ & $0,1946^{\mathrm{ns}}$ & $0,5840^{* *}$ & $0,3573^{\text {ns }}$ & $0,0048^{\mathrm{ns}}$ & $0,7222^{* *}$ & $0,8694^{* *}$ & $0,6975^{* *}$ & $0,6505^{* *}$ \\
\hline
\end{tabular}

(1)1, Iranduba, mata secundária, com adubação; 2, Iranduba, mata secundária, sem adubação; 3, Manaus, mata secundária, com adubação; 4, Manaus, mata secundária, sem adubação; 5, Manaus, capoeira, com adubação; 6, Manaus, capoeira, sem adubação; 7, Maués, mata primária, com adubação; 8, Maués, mata primária, sem adubação; 9, Maués, capoeira, com adubação; 10, Maués, capoeira, sem adubação. * e **Significativo a 5 e $1 \%$ de probabilidade,

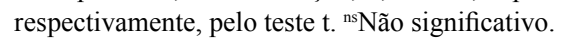


observadas para produção por planta entre os anos (Tabela 3), já que essa correlação é utilizada pelo método dos componentes principais (CPCOR) e pela análise estrutural de correlação (AECOR). Assim, o método CPCOV foi o mais indicado para estimar o coeficiente de repetibilidade em guaranazeiro. Esse resultado concorda com os obtidos por Cavalcanti et al. (2000) e Negreiros et al. (2008) em cajueiro-anão precoce e laranjeira-doce, respectivamente.

Os coeficientes de determinação foram baixos quando estimados pelos métodos ANOVA, CPCOR e
AECOR (Tabela 4). No método CPCOV, a média dos coeficientes de determinação foi de $80,44 \%$, com uma variação de 67,05 a $90,83 \%$.

Com base nos coeficientes de repetibilidade encontrados por meio dos quatro métodos, foi estimado o número de medições que devem ser realizadas para predizer o real valor dos clones, com base em coeficientes de determinação $\left(\mathrm{R}^{2}\right)$ pre-estabelecidos (Tabela 5). Calculando-se a média do número de medições entre os dezambientes para os quatro métodos, observou-se que haverá a necessidade de quatro $(80 \%$

Tabela 4. Coeficientes de repetibilidade $(r)$ e de determinação $\left(\mathrm{R}^{2}\right)$ da variável produção de sementes secas por planta, de clones de guaraná avaliados em diferentes ambientes, representados por solos caracterizados pelo tipo de vegetação capoeira, mata primária e mata secundária e em dois sistemas de cultivo - com e sem o uso de adubação - nos municípios de Manaus, Maués e Iranduba, no Estado do Amazonas, nos anos de 1998, 1999, 2000 e 2001.

\begin{tabular}{|c|c|c|c|c|c|c|c|c|}
\hline \multirow[t]{2}{*}{ Ambientes $^{(1)}$} & \multicolumn{2}{|c|}{ ANOVA } & \multicolumn{2}{|c|}{$\begin{array}{l}\text { Componentes principais } \\
\text { (Covariância) }\end{array}$} & \multicolumn{2}{|c|}{$\begin{array}{c}\text { Componentes principais } \\
\text { (Correlação) }\end{array}$} & \multicolumn{2}{|c|}{$\begin{array}{l}\text { Análise estrutural de } \\
\text { correlação (r médio) }\end{array}$} \\
\hline & $\mathrm{r}$ & $\mathrm{R}^{2}(\%)$ & $\mathrm{r}$ & $\mathrm{R}^{2}(\%)$ & $\mathrm{r}$ & $\mathrm{R}^{2}(\%)$ & $\mathrm{r}$ & $\mathrm{R}^{2}(\%)$ \\
\hline 1 & 0,1727 & 45,51 & 0,6136 & 86,40 & 0,2488 & 56,98 & 0,2202 & 53,04 \\
\hline 3 & 0,2581 & 58,19 & 0,3566 & 68,91 & 0,2817 & 61,07 & 0,2579 & 58,16 \\
\hline 4 & 0,1869 & 47,91 & 0,6002 & 85,73 & 0,2356 & 55,22 & 0,1212 & 35,55 \\
\hline 5 & 0,3423 & 67,55 & 0,4638 & 77,58 & 0,3768 & 70,75 & 0,3710 & 70,23 \\
\hline 6 & 0,6380 & 22,82 & 0,3976 & 72,53 & 0,1165 & 34,54 & 0,0744 & 24,32 \\
\hline 8 & 0,3300 & 66,33 & 0,7098 & 90,73 & 0,3349 & 66,82 & 0,2684 & 59,48 \\
\hline 9 & 0,4103 & 73,57 & 0,6951 & 90,12 & 0,4510 & 76,67 & 0,3962 & 72,41 \\
\hline 10 & 0,2520 & 57,40 & 0,7122 & 90,83 & 0,3348 & 66,82 & 0,3169 & 64,99 \\
\hline Médias $^{(2)}$ & 0,3183 & 56,41 & 0,5308 & 80,44 & 0,3064 & 59,39 & 0,2618 & 56,31 \\
\hline
\end{tabular}

(1)1, Iranduba, mata secundária, com adubação; 2, Iranduba, mata secundária, sem adubação; 3, Manaus, mata secundária, com adubação; 4, Manaus, mata secundária, sem adubação; 5, Manaus, capoeira, com adubação; 6, Manaus, capoeira, sem adubação; 7, Maués, mata primária, com adubação; 8, Maués, mata primária, sem adubação; 9 , Maués, capoeira, com adubação; 10 , Maués, capoeira, sem adubação. ${ }^{(2)}$ Média geral de $\mathrm{r}=0,3543$; média geral de $\mathrm{R}^{2}=63,14 \%$

Tabela 5. Número de anos de colheita associado a diferentes graus de determinação do valor genotípico $\left(\mathrm{R}^{2}\right)$ pelos métodos de estimação de repetibilidade ANOVA, CPCOR, CPCOV e AECOR, com base na variável produção anual de sementes secas por planta de clones de guaraná testados em solos caracterizados pelo tipo de vegetação capoeira, mata primária e mata secundária e em dois sistemas de cultivo - com e sem o uso de adubação - avaliados em diferentes ambientes durante quatro anos consecutivos $\left(1998,1999,2000\right.$ e 2001) nos municípios de Manaus, Maués e Iranduba, no Estado do Amazonas ${ }^{(1)}$.

\begin{tabular}{|c|c|c|c|c|c|c|c|c|c|c|c|c|}
\hline \multirow[t]{3}{*}{ Ambientes $^{(2)}$} & \multicolumn{12}{|c|}{ Número de medições para “Certos" $\mathrm{R}^{2}(\%)$} \\
\hline & \multicolumn{4}{|c|}{80} & \multicolumn{4}{|c|}{90} & \multicolumn{4}{|c|}{95} \\
\hline & ANOVA & CPCOR & CPCOV & AECOR & ANOVA & CPCOR & CPCOV & AECOR & ANOVA & CPCOR & $\mathrm{CPCOV}$ & AECOR \\
\hline 1 & 19,16 & 12,08 & 2,52 & 14,17 & 43,10 & 27,18 & 5,67 & 31,87 & 90,99 & 57,38 & 11,96 & 67,29 \\
\hline 2 & 11,92 & 7,85 & 7,86 & 11,48 & 26,82 & 17,65 & 17,69 & 25,84 & 56,61 & 37,27 & 37,35 & 54,54 \\
\hline 3 & 11,50 & 10,20 & 7,22 & 11,51 & 25,87 & 22,95 & 16,24 & 25,90 & 54,61 & 48,45 & 34,29 & 54,68 \\
\hline 4 & 17,40 & 12,98 & 2,66 & 29,01 & 39,14 & 29,19 & 5,99 & 65,28 & 82,63 & 61,63 & 12,66 & 137,81 \\
\hline 5 & 7,69 & 6,62 & 4,62 & 6,78 & 17,29 & 14,89 & 10,41 & 15,26 & 36,51 & 31,43 & 21,97 & 32,21 \\
\hline 6 & 54,12 & 30,32 & 6,06 & 49,78 & 121,77 & 68,23 & 13,64 & 112,01 & 257,07 & 144,03 & 28,79 & 236,46 \\
\hline 7 & 7,71 & 7,55 & 5,48 & 7,99 & 17,35 & 16,99 & 12,33 & 17,97 & 36,63 & 35,88 & 26,02 & 37,94 \\
\hline 8 & 8,12 & 7,95 & 1,64 & 10,90 & 18,27 & 17,88 & 3,68 & 24,53 & 38,57 & 37,74 & 7,77 & 51,78 \\
\hline 9 & 5,75 & 4,87 & 1,75 & 6,09 & 12,93 & 10,96 & 3,95 & 13,71 & 27,30 & 23,13 & 8,33 & 28,95 \\
\hline 10 & 11,88 & 7,95 & 1,62 & 8,62 & 26,72 & 17,88 & 3,64 & 19,39 & 56,41 & 37,74 & 7,68 & 40,95 \\
\hline Médias & 15,52 & 10,84 & 4,14 & 15,63 & 34,93 & 24,38 & 9,32 & 35,18 & 73,73 & 51,47 & 19,68 & 74,26 \\
\hline
\end{tabular}

${ }^{(1)}$ CPCOR, componentes principais com base na matriz de correlação; CPCOV, componentes principais com base na matriz de variâncias e covariâncias fenotípicas; e AECOR, análise estrutural com base na matriz de correlações ( $\mathrm{r}$ médio). (2)1, Iranduba, mata secundária, com adubação; 2 , Iranduba, mata secundária, sem adubação; 3, Manaus, mata secundária, com adubação; 4, Manaus, mata secundária, sem adubação; 5, Manaus, capoeira, com adubação; 6, Manaus, capoeira, sem adubação; 7, Maués, mata primária, com adubação; 8 , Maués, mata primária, sem adubação; 9, Maués, capoeira, com adubação; 10 , Maués, capoeira, sem adubação. 
de acurácia, CPCOV) a 74 anos (95\% de acurácia, AECOR) de avaliação efetiva (Tabela 5). O método CPCOV foi o mais eficiente em determinar o período mínimo de avaliações da produção no guaranazeiro. Os métodos ANOVA e AECOR apresentaram os maiores valores para o período mínimo de avaliações efetivas, independentemente da acurácia, o que os torna impraticáveis para utilização no programa de melhoramento do guaranazeiro.

Deve-se considerar que as estimativas de repetibilidade não só variam em relação à natureza da característica que está sendo analisada, mas também em relação às propriedades genéticas da população e das condições às quais os indivíduos são submetidos (Cruz et al., 2004). Isso pode ser verificado no ambiente 6, considerado desfavorável para a obtenção da predição do real valor dos clones com uma acurácia de no mínimo $80 \%$, conforme os resultados das análises pelos métodos ANOVA, CPCOR e AECOR (Tabela 5). Segundo Escobar (1986), a longevidade da cultura é de aproximadamente 30 anos e haveria a necessidade de avaliação durante toda a vida útil das plantas, pois, nas condições do Estado do Amazonas, a produção ocorre apenas uma vez por ano, o que seria inviável. Nesse caso, uma estratégia que o programa de melhoramento genético do guaranazeiro poderia adotar para obter o número mínimo de medidas necessárias para a obtenção do real valor dos clones com maior acurácia seria submetê-los a ambientes favoráveis, a exemplo do ambiente 7 .

\section{Conclusões}

1. As estimativas dos coeficientes de repetibilidade para o caráter produção de sementes de guaraná são relativamente baixas e variam de acordo com as metodologias e ambientes.

2. São necessários no mínimo quatro anos de avaliação para a obtenção de valores genotípicos dos clones com $\mathrm{R}^{2}$ de $80 \%$.

\section{Referências}

ABEYWARDENA, V. An application of principal component analysis in genetics. Journal of Genetics, v.61, p.27-51, 1972.

ATROCH, A.L.; NASCIMENTO FILHO, F.J. do. Classificação do coeficiente de variação na cultura do guaranazeiro. Revista de Ciências Agrárias, n.43, p.43-48, 2005.
BONOMO, P. Metodologias biométricas para seleção de progênies no melhoramento genético do cafeeiro. 2002. 124p. Tese (Doutorado) - Universidade Federal de Viçosa, Viçosa.

CARVALHO, C.G.P. Repetibilidade e seleção de híbridos de cacaueiro. 1999. 176p. Tese (Doutorado) - Universidade Federal de Viçosa, Viçosa.

CAVALCANTI, J.J.V.; PAIVA, J.R. de; BARROS, L. de M.; CRISÓSTOMO, J.R.; CORRÊA, M.P.F. Repetibilidade de caracteres de produção e porte da planta em clones de cajueiro-anão precoce. Pesquisa Agropecuária Brasileira, v.35, p.773-777, 2000.

CEDILLO, D.S.O. Análises biométricas aplicadas ao melhoramento de dendê (Elaeis guineensis, Jacq). 2003. 94p. Dissertação (Mestrado) - Universidade Federal de Viçosa, Viçosa.

CHAPMAN, A.B. General and quantitative genetics. Amsterdam: Elsevier, 1985. 408p.

CORNACCHIA, G.; CRUZ, C.D.; LOBO, P.R.; PIRES, I.E. Estimativas do coeficiente de repetibilidade para características fenotípicas de procedências de Pinus tecunumanii (Schw.) Eguiluz e Perry e Pinus caribaea var. hondurensis Barret, Golfari. Revista Árvore, v.19, p.333-345, 1995.

COSTA, J.G. da; LEDO, A.S.; OLIVEIRA, M.N. Estimativas de repetibilidade de características de frutos de cupuaçuzeiro no Estado do Acre. Revista Brasileira de Fruticultura, v.19, p.313-318, 1997.

CRUZ, C.D. Programa GENES: versão Windows: aplicativo computacional em genética e estatística. Viçosa: UFV, 2001. $648 \mathrm{p}$.

CRUZ, C.D.; REGAZZI, A.J.; CARNEIRO, P.C.S. Modelos biométricos aplicados ao melhoramento genético. 3.ed. Viçosa: UFV, 2004. 480p.

DIAS, L.A.S.; SOUSA, C.A.S. Aplicação do coeficiente de repetibilidade na seleção de cacaueiros em plantação comercial. Revista Brasileira de Genética, v.16, p.364, 1993.

ESCOBAR, J.R. Herdabilidade de alguns caracteres da fase juvenil de clones de guaraná (Paullinia cupana var. sorbilis). Manaus: Embrapa-Uepae, 1986. 23p. (Embrapa/Uepae. Boletim de Pesquisa, 6).

ESCOBAR, J.R.; CORREA, M.P.F.; BARRETO, J.F. Estimativa do número de folhas e ramos, altura de planta, tamanho de amêndoa e produção de guaraná. In: SIMPÓSIO BRASILEIRO DO GUARANÁ, 1., 1983, Manaus. Anais. Manaus: Embrapa-Uepae de Manaus, 1984. (Embrapa-Uepae de Manaus. Documentos, 3).

FALCONER, D.S. Introdução à genética quantitativa. Viçosa: UFV, 1981. 279p.

FALCONER, D.S. Introduction to quantitative genetics. New York: Longman, 1989. 438p.

FERREIRA, R. de P.; BOTREL, M. de A.; PEREIRA, A.V.; CRUZ, C.D. Avaliação de cultivares de alfafa e estimativas de repetibilidade de caracteres forrageiros. Pesquisa Agropecuária Brasileira, v.34, p.995-1002, 1999.

GONÇALVES, P. de S.; CARDOSO, M.; SAES, L.A. Estimativas de repetibilidade na seleção de árvores adultas de seringueira. Pesquisa Agropecuária Brasileira, v.25, p.1031-1038, 1990. 
KEMPTHORNE, O. An introduction to genetic statistics. 2.ed. Ames: Iowa State University Press, 1973. 545p.

LOPES, R.; BRUCKNER, C.H.; CRUZ, C.D.; LOPES, M.T.G.; FREITAS, G.B. de. Repetibilidade de características do fruto de aceroleira. Pesquisa Agropecuária Brasileira, v.36, p.507-513, 2001.

LUSH, J.L. Melhoramento dos animais domésticos. Rio de Janeiro: Cedegra, 1964. 570p.

MORRISON, D.F. Multivariate statistical methods. New York: McGraw-Hill, 1967. 415p.

MANSOUR, H.; NORDHEIM, E.V.; RUTLEDGE J.J. Estimators of repeatability. Theoretical and Applied Genetics, v.60, p.151-156, 1981.

NASCIMENTO FILHO, F.J. do; ATROCH, A.L.; CRAVO, M. da S. Melhoramento genético do guaranazeiro: resultados de ensaios de avaliação de clones: fase produtiva 1985 a 1994. Manaus: Embrapa Amazônia Ocidental, 2000a. 54p. (Embrapa Amazônia Ocidental. Boletim de Pesquisa, 7).

NASCIMENTO FILHO, F.J. do; ATROCH, A.L.; CRAVO, M. da S.; GARCIA, T.B.; RIBEIRO, J. de R.C.; LIMA, L. dos P.; FERREIRA, J.O. Novos clones de guaranazeiro para o Estado do Amazonas. Manaus: Embrapa Amazônia Ocidental, 2000b. 3p. (Embrapa Amazônia Ocidental. Comunicado Técnico, 8).

NEGREIROS, J.R. da S.; SARAIVA, L.L.; OLIVEIRA, T.K. de; ÁLVARES, V. de S.; RONCATTO, G. Estimativas de repetibilidade de caracteres de produção em laranjeiras-doces no Acre. Pesquisa Agropecuária Brasileira, v.43, p.1763-1768, 2008.
PEREIRA, A.V.; FERREIRA, R.P.; CRUZ, C.D.; FREITAS, V.P.; OLIVEIRA, P.T.A. Comportamento da alfafa cv. Crioula de diferentes origens e estimativas dos coeficientes de repetibilidade para caracteres forrageiros. Revista Brasileira de Zootecnia, v.27, p.686-690, 1998.

RESENDE, M.D.V. de; STURION, J.A.; MENDES, S. Genética e melhoramento de erva-mate (Ilex paraguariensis St. Hil). Colombo: Embrapa-CNPF, 1995. 33p. (Embrapa-CNPF. Documentos, 25).

RUTLEDGE, J.J. Scaling which removes bias of Abeywardenas estimator of repeatability. Journal of Genetics, v.61, p.247-250, 1974.

SIQUEIRA, E.R. Coeficiente da repetibilidade da produção de frutos de coqueiro comum. Pesquisa Agropecuária Brasileira, v.17, p.573-574, 1982.

SMYTH, T.J.; CRAVO, M.S. Resposta do guaranazeiro a níveis de N, P, K e Mg. Manaus: Embrapa Amazônia Ocidental, 1989. (Embrapa Amazônia Ocidental. Relatório de Projeto).

SOARES, E.B.; GOMES, R.L.F.; CAMPELO, J.E.G.; LOPES, A.C. de A.; MATOS FILHO, C.H.A. Repetibilidade e correlações entre caracteres morfo-agronômicos de cajazeira. Ciência e Agrotecnologia, v.32, p.1851-1857, 2008.

TURNER, H.N.; YOUNG, S.S.Y. Quantitative genetics in sheep breeding. New York: Cornell University, 1969. 332p.

VALLOIS, A.C.C.; CORRÊA, M.P.F.; VASCONCELLOS, M.E.C. Estudo de caracteres correlacionados com a produção de amêndoa seca no guaranazeiro. Pesquisa Agropecuária Brasileira, v.14, p.175-179, 1979.

Recebido em 26 de janeiro de 2009 e aprovado em 29 de maio de 2009 